EPJ Web of Conferences 47, 05010 (2013)

DOI: $10.1051 /$ epjconf/20134705010

(C) Owned by the authors, published by EDP Sciences, 2013

\title{
Advances in precision Doppler spectroscopy on cool stars
}

\author{
Guillem Anglada-Escudéa \\ Universität Göttingen, Institut für Astrophysik, Friedrich-Hund-Platz 1, 37077 Göttingen, \\ Germany
}

\begin{abstract}
I describe recent advances made in Doppler spectroscopy of low mass star and discuss how they perform on public observations ontained with the HARPS spectrograph. This work is possible thanks to the recent development of the HARPS-TERRA software (Template Enhanced Radial velocity Re-analysis Application), which obtains precision RV measurements by least-squares matching each spectrum to a high SNR template built from the same observations. As a result, we obtain a substantial increase in precision compared to the traditional cross-correlation methods. The increase in precision is demonstrated with RV measurements on stable M dwarfs $(80 \mathrm{~cm} / \mathrm{s}$ over time-scales of years) and the early detection of several very low mass candidates. Moreover, the ability of HARPS-TERRA in reproducing the observed spectra at high fidelity allows us to explore new effects including : wavelength dependence of activity induced Doppler signals, simultaneous measurement of orbital obliquities and stellar rotation profiles and, when combined with advanced Bayesian data analysis techniques, small Doppler signals likely caused by new super-Earth mass candidates in the habitable zones of nearby stars.
\end{abstract}

Continous technical improvements in the last 15 years has allowed the Doppler spectroscopic technique to reach precisions at the $1 \mathrm{~m} \mathrm{~s}^{-1}$ level on $\mathrm{G}$ and $\mathrm{K}$ dwarfs. Although low mass stars (M dwarfs) are very rich in spectral features and Doppler information, none had been identified to be as stable as the most quiet $\mathrm{K}$ and $\mathrm{G}$ dwarfs. In an effort to investigate this issue, we developed the TERRA software [Template Enhanced Radial velocity Re-analysis Application, 1] and apply it to HARPS public observations of nearby $M$ dwarfs. The method consists on building a very high S/R template of the spectra by coadding all the available observations and then measure the Doppler offset of each spectra using a simple least-squares matching algortithm. Compared the the cross-correlation function method (CCF) implemented by the HARPS-ESO Data Reduction Software, TERRA requires no prior assumption on the nature of the star and is able to use every single feature in the stellar spectra.

Spectra of M dwarf at optical wavelengths are completely dominated by molecular absorption features with severe blending of hundreds of lines (see Fig. 1). The digital CCF method was developed in the 90' to deal primarily with solar-like stars and relies on using well isolated lines to centroid an effective mean line profile. While for $\mathrm{G}$ and $\mathrm{K}$ dwarfs CCF and TERRA achive similar performance, and the improvement in precision is very notable in $\mathrm{M}$ dwarfs (typically, it removes about $1-2 \mathrm{~m} \mathrm{~s}^{-1}$ systematic noise jitter). As a result, small low mass companions can be detected significantly earlier (with about 20-30\% of the observations) and/or smaller amplitudes can be investigated.

TERRA also allows independent verification of signals and a new suit of test to validate planet candidates. For example, we have investigated the wavelength dependence of Doppler signals finding that most stars show substantial random variability towards the blue (theoretically expected, but never proved before on low activity stars). We also found that some of this variability is not random and typically correlated with chromospheric emission (S-index) at time-scales of 100 days or more $[1,2]$.

\footnotetext{
ae-mail: guillem.anglada@gmail.com
}

This is an Open Access article distributed under the terms of the Creative Commons Attribution License 2.0, which permits unrestricted use, distribution, and reproduction in any medium, provided the original work is properly cited. 


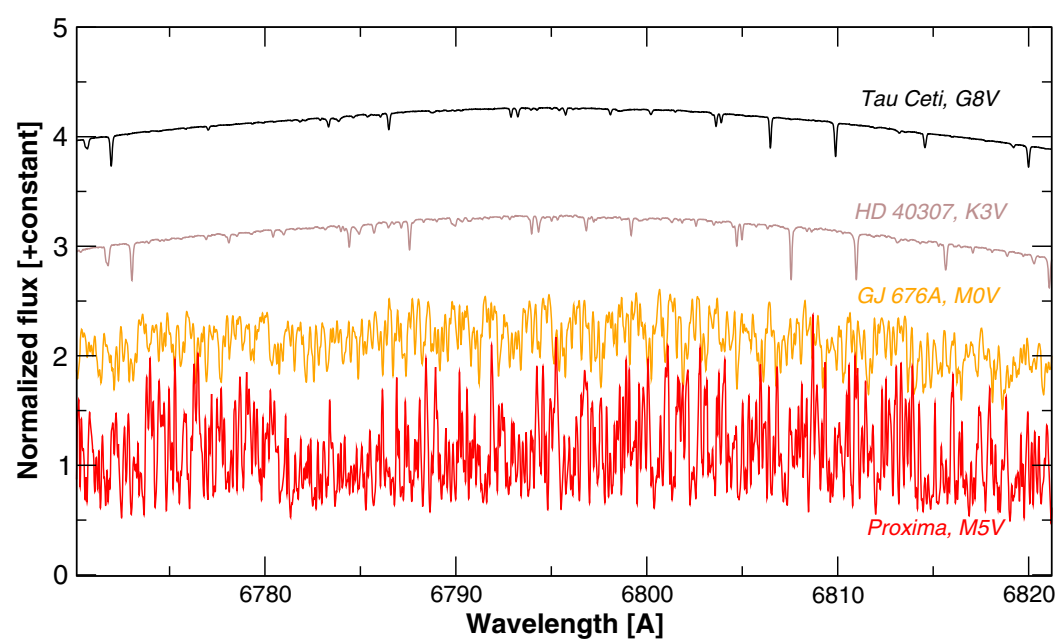

Figure 1. Coadded spectra of a G, K, M0 and mid-M dwarfs at the 70th Echelle apperture of HARPS. All spectra have SNR larger than 200 and, therefore, all the features in the last two spectra are real. The extreme amount of features on the spectra of the M dwarfs makes the CCF technique (requires selecting isolated lines) to work sub-optimally.

Table 1. Overview of systems where HARPS-TERRA velocities have been used to report new candidates.

\begin{tabular}{llll}
\hline Star & Sp.Type & New planet candidates & Ref. \\
\hline \hline GJ 667C & M2V & 2 (possibly 3) super-Earths & {$[7]$} \\
GJ 676A & M0V & 1 super-Earth, 1 Neptune & {$[5]$} \\
HD 40307 & K3V & 3 super-Earth & {$[2]$} \\
NewStar1 & K7V & 1 super-Earth, 1 sub-Saturn & {$[9]$} \\
NewStar2 & M & up to 7 super-Earths & {$[10]$} \\
\hline
\end{tabular}

The combination of these techniques allow filtering some of the activity induced Doppler variability and identify new low mass planet candidates. For example, HARPS-TERRE measurements together combined with the Bayesian techniques developed by M. Tuomi [e.g., 3], enabled the removal of the stellar activity cycle and the detection of 6 super-Earth candidates around HD 40307 - three more than those reported before [4]- one of them within the habitable zone of the star ( $\mathrm{P} \sim 200$ days, $\left.\mathrm{M} \sin \mathrm{i} \sim 7 \mathrm{M}_{\text {earth }}\right)$. Similar techniques have been used to detect two more low mass candidates around GJ 676A [5] where two gas giants had already been reported before [6], the system around GJ 667C $[7,8]$ with one super-Earth in its habitable zone, and a few more forthcoming announcements (see Table 1). This detection results indicate that a significant number of low mass stars $(\sim>10 \%)$ are overabundant in multiple low mass planets.

\section{CONCLUSIONS}

Template matching techniques (and forward modeling in general) applied to stabilized spectrographs have the potential to open a whole set of new science applications (e.g., measure of stellar rotation profiles when a planet is transting at the same time as the orbital obliquity).

The ability of reproducing high-precision measurements and the new tests we have developed provide an important validation tool for recently announced very low mass amplitude candidates [e.g., $\alpha \mathrm{CenBb}$ and several others 11,12$]$. Given that such high precision meausements are almost impossible to reproduce (e.g., very high over-subscription rates for new programs with HARPS), we insist that open 


\section{Hot Planets and Cool Stars}

data policies (including release of raw data) should be implemented as soon as such oustanding results are announced.

\section{References}

[1] G. Anglada-Escudé, R.P. Butler, ApJS 200, 15 (2012), 1202. 2570

[2] M. Tuomi, G. Anglada-Escudé, E. Gerlach, H.R.A. Jones, A. Reiners, E.J. Rivera, S.S. Vogt, R.P. Butler, A\&A 549, A48 (2013), 1211.1617

[3] M. Tuomi, A\&A 543, A52 (2012), 1204.1254

[4] M. Mayor, X. Bonfils, T. Forveille, X. Delfosse, S. Udry, J. Bertaux, H. Beust, F. Bouchy, C. Lovis, F. Pepe et al., A\&A 507, 487 (2009), 0906. 2780

[5] G. Anglada-Escudé, M. Tuomi, A\&A 548, A58 (2012), 1206.7118

[6] T. Forveille, X. Bonfils, G. Lo Curto, X. Delfosse, S. Udry, F. Bouchy, C. Lovis, M. Mayor, C. Moutou, D. Naef et al., A\&A 526, A141+ (2011), 1012.1168

[7] G. Anglada-Escudé, P. Arriagada, S.S. Vogt, E.J. Rivera, R.P. Butler, J.D. Crane, S.A. Shectman, I.B. Thompson, D. Minniti, N. Haghighipour et al., ApJL 751, L16 (2012), 1202.0446

[8] X. Delfosse, X. Bonfils, T. Forveille, S. Udry, M. Mayor, F. Bouchy, M. Gillon, C. Lovis, V. Neves, F. Pepe et al., arXiv:1202.2467 (2012), 1202.2467

[9] P. Arriagada, G. Anglada-Escudé, R.P. Butler, et al., submitted to A\&A (2012)

[10] G. Anglada-Escudé, M. Tuomi, E. Gerlach, R. Heller, R. Barnes, J. Jenkins, R.P. Butler, H.R.A. Jones, S.S. Vogt, submitted to A\&A (2013)

[11] X. Dumusque, F. Pepe, C. Lovis, D. Ségransan, J. Sahlmann, W. Benz, F. Bouchy, M. Mayor, D. Queloz, N. Santos et al., Nature 491, 207 (2012)

[12] F. Pepe, C. Lovis, D. Ségransan, W. Benz, F. Bouchy, X. Dumusque, M. Mayor, D. Queloz, N.C. Santos, S. Udry, A\&A 534, A58+(2011), 1108.3447 